\title{
Novel Architecture of Content Delivery Network Based on Big Data for Power Saving
}

\author{
Tae-Kook Kim \\ Department of Information, Communications \& Software Engineering, Tongmyong University, \\ Busan, Republic of Korea \\ leader@tu.ac.kr
}

Received Date : November 09, 2021 Accepted Date : November 29, 2021 Published Date : December 07, 2021

\begin{abstract}
This paper proposes an architecture of content delivery network (CDN) based on big data for power saving. There are two types of video content: hot content and cold content. When video content is accessed frequently, it is called hot content. Conversely, when video content is accessed infrequently, it is called cold content. In CDN, there is an origin server and a CDN cache server. A CDN cache server has a replicated content and provides its content to the end users nearby. Therefore, the user can receive the requested content from the closest proximity for fast content. The proposed architecture in this paper powers off the cold content server in CDN cache server when the number of cold content requests decreases. Hence, the proposed architecture for content delivery services based on power saving is expected to be useful for providing multimedia streaming services with low power consumption for content providers.
\end{abstract}

Key words : content delivery network (CDN), content distribution, big data, Artificial Intelligence (AI)

\section{INTRODUCTION}

With the spread of smartphones and tablets, network traffic such as video content is increasing rapidly these days. In order to process such a large amount of video traffic, content delivery network (CDN) technology is required [1-4].

Content delivery network is a technology that can provide content more quickly from a content provider (ex. YouTube, Netflix) to users who are geographically and physically separated from each other. CDN consists of an origin server and a CDN cache server (surrogate server). The origin server has all the original content. And in general, the CDN cache server has a copy of all the content of the origin server. There is a problem of additional power consumption due to the use of a replicated server (CDN cache server). The CDN cache server requires additional power to operate, and this enables to provide fast content delivery to users. Hence, there is a need for research on an efficient content delivery network technology to reduce power consumption [5-8].

To solve this problem, I proposed the architecture of content delivery network based on big data for power saving. The proposed architecture turns off the cold content server of the $\mathrm{CDN}$ cache server when it recognizes the reduced number of user requests. When video content is accessed infrequently, it is called cold content. The requested cold content is provided by the origin server. This can reduce the power consumption of the CDN cache server.The decrease in user requests can be recognized using big data technology. By analyzing the request pattern of users, it is possible to analyze the days and times when requests are reduced.

This study is organized as follows. Chapter 2 briefly explains the backgrounds. The proposed architecture of CDN based on big data for power saving is described in chapter 3. Lastly, chapter 4 presents the conclusion.

\section{BACKGROUNDS}

\subsection{Content Delivery Network}

Content delivery network is a technology that stores (caching) the content in a cache server located close to the user and responds to the content request by the cache server. Basically, when a user downloads content (ex. Web Object, Video, Music, Image, Document, etc.) from a remote server (Origin Server), it takes longer than a server nearby $[5,6,9,10]$.

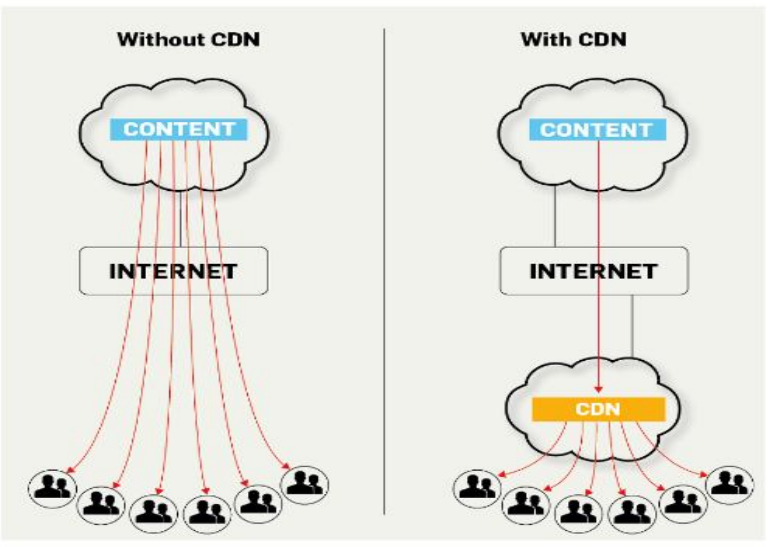

Figure 1: Architecture of $\mathrm{CDN}$ (without $\mathrm{CDN}$ vs. with $\mathrm{CDN}$ ) 
Tae-Kook Kim, International Journal of Emerging Trends in Engineering Research, 9(12), December 2021, 1508 - 1511

Figure 1 shows the architecture of the CDN. This figure shows the comparison between without $\mathrm{CDN}$ and with CDN. The CDN cache server has the same content data on the origin server. Therefore, there are problems such as an increase in operating cost and power consumption due to the operation of the CDN cache server [11-13].

\subsection{Hot Content and Cold Content}

There are two types of video content such as hot content and cold content. When video content is accessed frequently, it is called hot content. Conversely, when video content is accessed infrequently, it is called cold content. The origin server stores all hot and cold content. And in general, the CDN cache server has a copy of all the content (hot content, cold content) of the origin server $[9,10]$.

\section{PROPOSED ARCHITECTURE OF CDN BASED ON BIG DATA FOR POWER SAVING}

This paper proposed architecture of CDN based on big data for power saving. The proposed architecture turns off the cold content server of the $\mathrm{CDN}$ cache server when it recognizes the reduced number of user requests. And the requested cold content is provided by the origin server. In this manner, you can reduce the power consumption of the $\mathrm{CDN}$ cache server. The decrease in user requests can be recognized using big data technology. By analyzing the request pattern of users, it is possible to analyze the days and times when requests are reduced. In this study, it was assumed that the decrease in requests for cold content could be recognized through big data analysis.

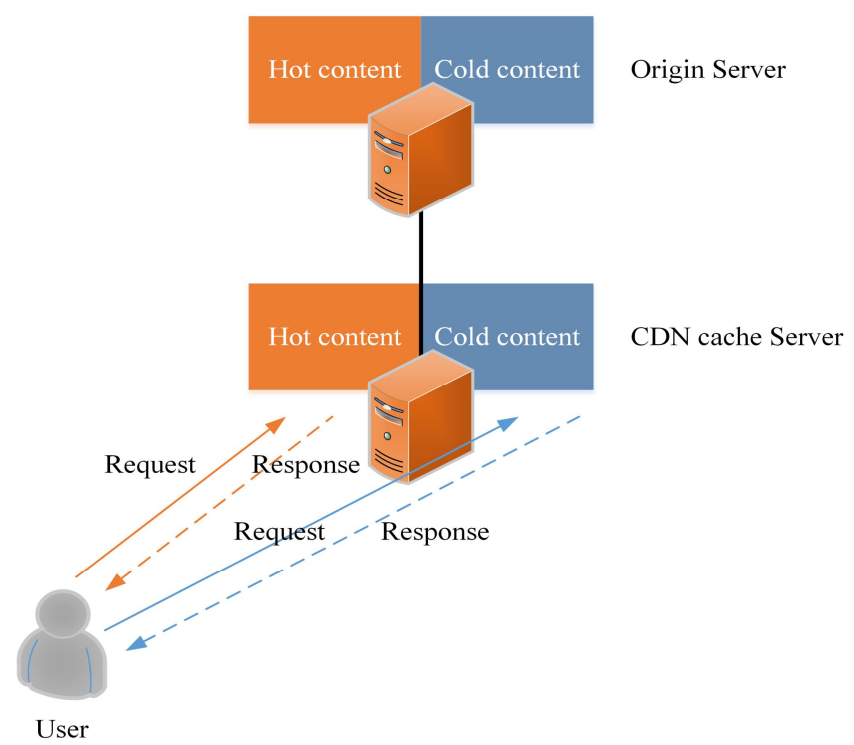

Figure 2: Conventional architecture of CDN

Figure 2 shows the conventional architecture of CDN. Assuming that all content of origin server is copied to CDN cache server, user's content request is processed by CDN cache server. The CDN cache server also stores all content data. Therefore, the CDN cache server requires a large amount of power to provide content delivery service.

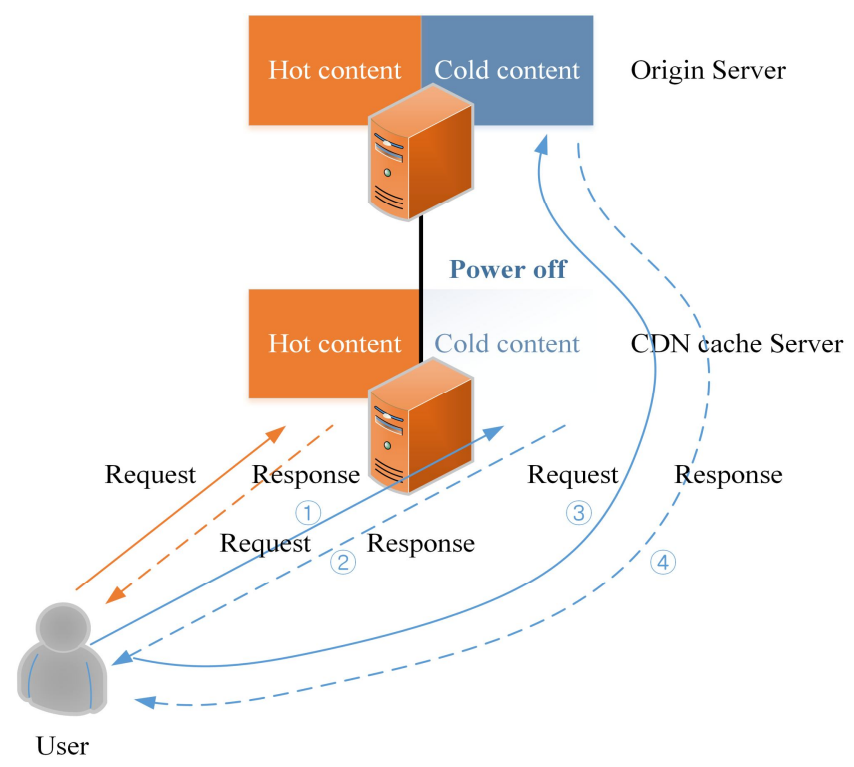

Figure 3: Proposed architecture of CDN

Figure 3 shows the proposed architecture for content delivery services. Both hot and cold content are copied to the CDN cache server. Hot content is a popular content that is frequently requested. On the other hand, cold content is an unpopular content that is sometimes requested. When cold content requests are drastically reduced (ex. dawn), the cold content server of the CDN cache server is turned off. And the requested cold content is provided by the origin server.

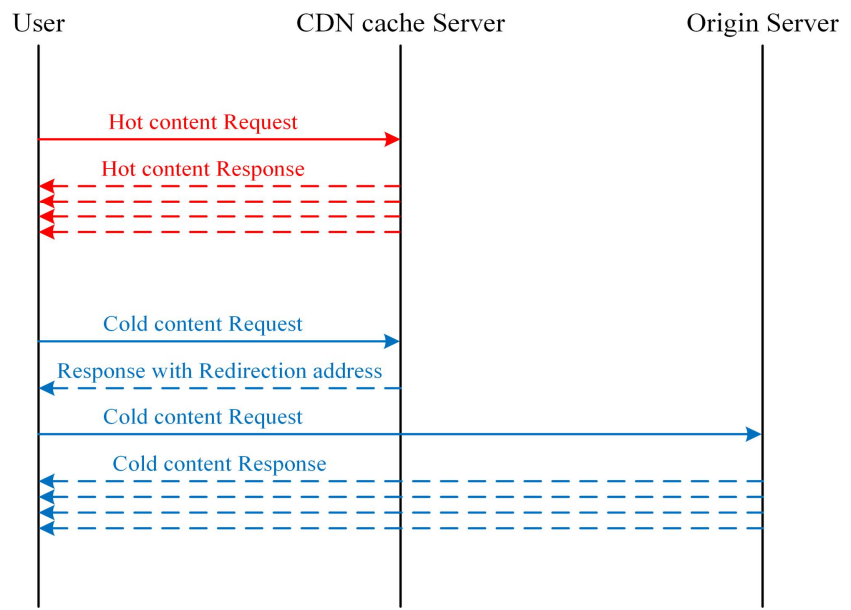

Figure 4: Flow chart of proposed architecture of CDN (shut down of cold content server of the CDN cache servers)

Figure 4 shows the flow chart of the proposed architecture of $\mathrm{CDN}$ for cold content request. When there are few requests for cold content based on big data, shut down the cold content server of the CDN cache server. In this way, power consumption can be reduced. A user requests cold content from the CDN cache server. Since the cold content server of 
Tae-Kook Kim, International Journal of Emerging Trends in Engineering Research, 9(12), December 2021, 1508 - 1511

the CDN cache server is powered off, it informs the origin server address. Through this redirection, users can download cold content from the origin server.

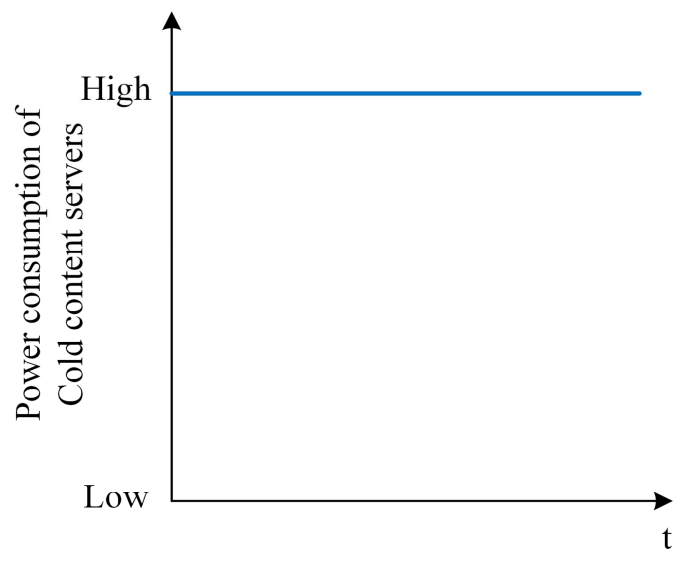

(a) weight factor $\alpha=1$

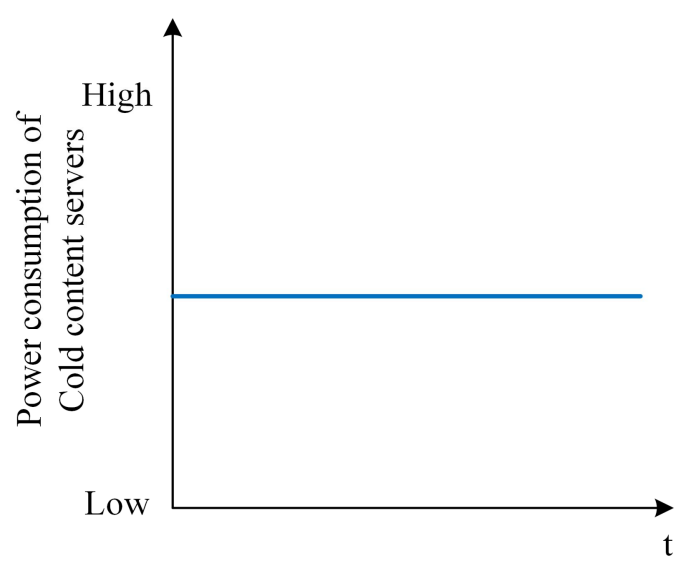

(b) weight factor $\alpha=0.5$

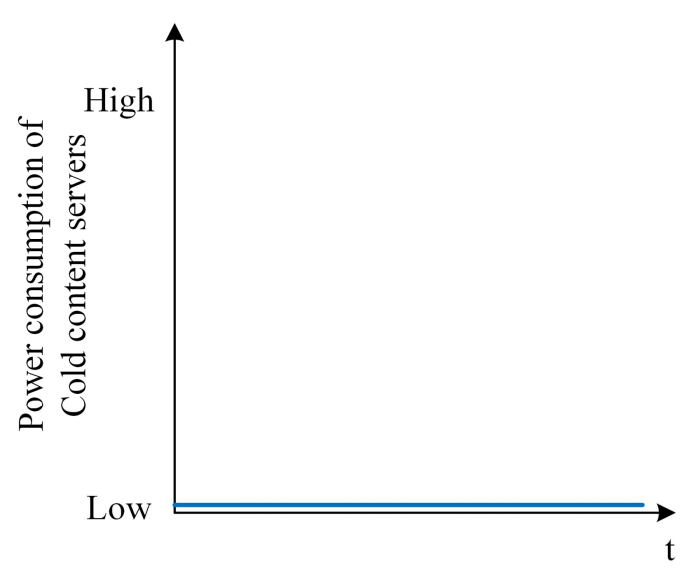

(c) weight factor $\alpha=0$

Figure 5: Power consumption of cold content servers (weight factor $\alpha=0, \alpha=0.5, \alpha=1)$

$\mathrm{C}=\alpha \cdot \mathrm{C}_{\text {power(cold_content) }}+(1-\alpha) \mathrm{C}_{\text {delay(cold_content) }}$

where $\mathrm{C}$ is cost, $\alpha$ is weight factor, $\mathrm{C}_{\text {power(cold_content) }}$ is the cost of power consumption, and $\mathrm{C}_{\text {delay(cold_content) }}$ is the cost of delay.

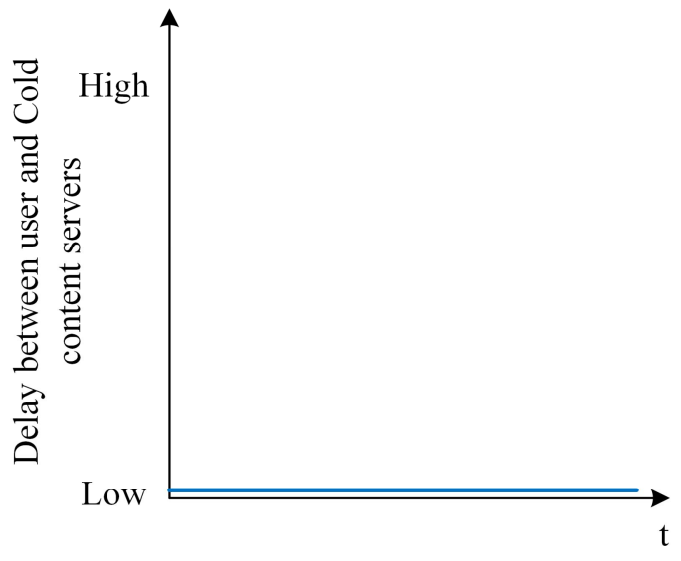

(a) weight factor $\alpha=1$

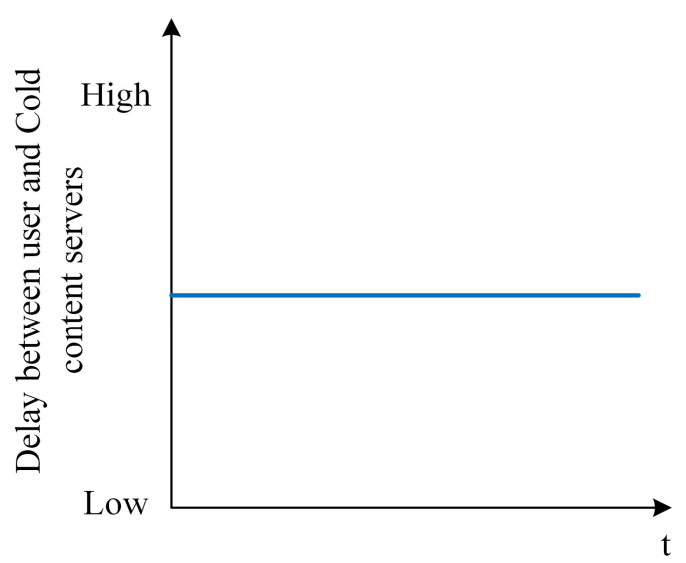

(b) weight factor $\alpha=0.5$

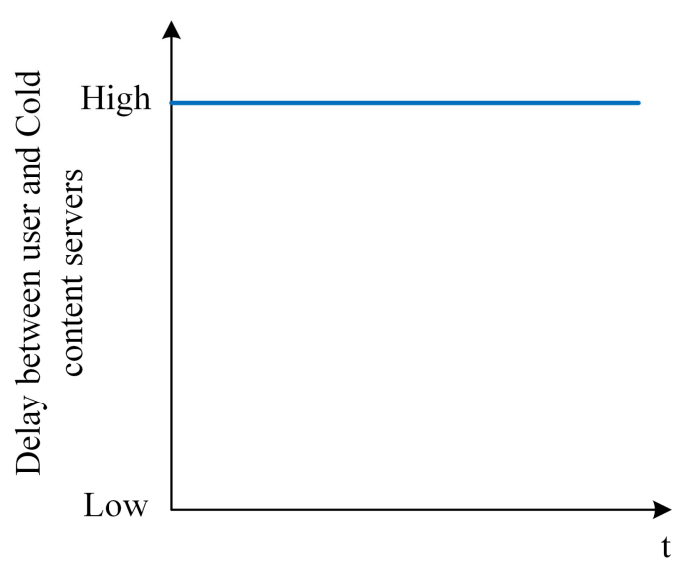

(c) weight factor $\alpha=0$

Figure 6: Delay between user and cold content servers (weight factor $\alpha=0, \alpha=0.5, \alpha=1$ )

Figure 5 shows the power consumption according to the value of $\alpha$. When the weight factor $\alpha$ value is 1 , the same power as conventional architecture of $\mathrm{CDN}$ is consumed. Although the $\mathrm{CDN}$ cache server consumes a lot of power, the transmission delay of cold content can be reduced. This is because the service is provided by the CDN cache server near the user 
Tae-Kook Kim, International Journal of Emerging Trends in Engineering Research, 9(12), December 2021, 1508 - 1511

rather than the remote origin server.

When the value of weight factor $\alpha$ is 0 , the power of the cold content server of the CDN cache server is turned off and cold content is transmitted from the origin server. Although power consumption can be reduced, the delay in cold content transmission increases as figure 6 . This is because the service is provided by the remote origin server rather than the CDN cache server near the user.

Figure 6 shows the delay according to the value of $\alpha$. When the weight factor $\alpha$ value is 1 , the same delay as the existing $\mathrm{CDN}$ architecture is indicated. Although the transmission delay of cold content can be reduced, the CDN cache server consumes a lot of power. This is because the service is provided by the CDN cache server near the user rather than the remote origin server.

When the value of weight $\alpha$ is 0 , cold content is served by the origin server. Therefore, there is a delay in the transmission of the cold content. This is because the service is provided by the remote origin server rather than the $\mathrm{CDN}$ cache server near the user. It is possible to optimize power consumption and delay by adjusting the weight factor $\alpha$ value according to time or network situation. For example, the tradeoff between power consumption and delay can be adjusted by setting the $\alpha$ value to 0.5 or the like.

The architecture proposed in this paper turns off the power of the cold content server when the number of cold content requests from the $\mathrm{CDN}$ cache server decreases based on big data. It shuts down the cold content server of the CDN cache server and receives content from the origin server. Hence, the proposed architecture for content delivery services based on big data is expected to be useful for providing multimedia streaming services with low power consumption for content providers.

\section{CONCLUSION}

In this study, we present the architecture of CDN based on big data for power saving. The proposed architecture has the advantage of reducing power consumption. If it detects the decreased content request, the cold content server is turned off in $\mathrm{CDN}$ cache server. The cold content with less user requests is provided by the origin server. In this manner, the power of the CDN cache server can be reduced. Therefore, the proposed architecture of content delivery services is expected to be useful for content provider with low power consumption.

\section{ACKNOWLEDGEMENT}

This Research was supported by the Tongmyong University Research Grants 2018 (2018A013).

\section{REFERENCES}

1. Swetha, M., \& Prakash, D. R.. Arrangement and Popularity Busting of YouTube Videos of Effected
Related Data, International Journal of Advanced Science and Technology, Vol. 28, No. 7, pp. 22-28, 2019

2. Kim, T. K.. A mobile multimedia content handoff scheme based on proxy mobile IPv6 for VR/AR, Multimedia Tools and Applications, Vol. 79, No. 23, pp. 16501-16515, 2020.

3. Quan Yuan, Haibo Zhou, Jinglin Li, Zhihan Liu, Fangchun Yang, Xuemin Sherman Shen. Toward efficient content delivery for automated driving services: An edge computing solution, IEEE Network, Vol. 32, No.1, pp. 80-86, 2018.

4. Ling Liu, Yiqing Zhou, Jinhong Yuan, Weihua Zhuang, Ying Wang. Economically optimal MS association for multimedia content delivery in cache-enabled heterogeneous cloud radio access networks, IEEE Journal on Selected Areas in Communications, Vol. 37, No.7, pp. 1584-1593, 2019.

5. Kim, Taekook, Eui-Jik Kim. Hybrid storage-based caching strategy for content delivery network services, Multimedia Tools and Applications, Vol. 74, No.5, pp. 1697-1709, 2015.

6. Kim, Taekook, Eui-Jik Kim. View pattern-based adaptive streaming strategy for mobile content delivery services, Multimedia Tools and Applications, Vol. 75, No.20, pp. 12693-12704, 2016.

7. Huy Nguyen T., Hoang Duong Tuan, Trung Q. Duong, H. Vincent Poor, Won-Joo Hwang. Collaborative multicast beamforming for content delivery by cache-enabled ultra dense networks, IEEE Transactions on Communications, Vol. 67, No.5, pp. 3396-3406, 2019.

8. Liang Zhou, Dan Wu, Zhenjiang Dong, Xuelong Li. When collaboration hugs intelligence: Content delivery over ultra-dense networks, IEEE Communications Magazine, Vol. 55, No.12, pp. 91-95, 2017.

9. Kim, Taekook, Li Chunying, Yim Taihyong, Kim, Youngjun, Kim Myeongyu, Park, Jinwoo. Novel architecture for a mobile content delivery network based on proxy mobile IPv6, IEICE Transactions on Fundamentals of Electronics, Communications and Computer Sciences Vol. 97.3, pp. 907-910, 2014.

10. Kim, Tae-Kook, Jung-Hyok Kwon, Eui-Jik Kim. Categorization-based video streaming for traffic mitigation in content delivery services, Multimedia Tools and Applications, Vol. 76, No. 23, pp. 25495-25510, 2017.

11. Vakali, Athena, George Pallis. Content delivery networks: Status and trends, IEEE Internet Computing, Vol.7, No. 6, pp. 68-74, 2003.

12. Akamai,

https://www.akamai.com/solutions/content-delivery-net work/

13. Buyya, Rajkumar, Mukaddim Pathan, and Athena Vakali, eds. Content delivery networks. Vol. 9. Springer Science \& Business Media, 2008. 